\title{
Microscopic dynamics underlying the anomalous diffusion
}

\author{
G. Kaniadakis ${ }^{1 *}$, G. Lapenta ${ }^{2 \dagger}$ \\ Istituto Nazionale per la Fisica della Materia - Unità del Politecnico di Torino \\ ${ }^{1}$ Dipartimento di Fisica and ${ }^{2}$ Dipartimento di Energetica - Politecnico di Torino \\ Corso Duca degli Abruzzi 24, 10129 Torino, Italy
}

(October 31, 2018)

The time dependent Tsallis statistical distribution describing anomalous diffusion is usually obtained in the literature as the solution of a non-linear Fokker-Planck (FP) equation [A.R. Plastino and A. Plastino, Physica A, 222, 347 (1995)]. The scope of the present paper is twofold. Firstly we show that this distribution can be obtained also as solution of the non-linear porous media equation. Secondly we prove that the time dependent Tsallis distribution can be obtained also as solution of a linear FP equation [G. Kaniadakis and P. Quarati, Physica A, 237, 229 (1997)] with coefficients depending on the velocity, that describes a generalized Brownian motion. This linear FP equation is shown to arise from a microscopic dynamics governed by a standard Langevin equation in presence of multiplicative noise.

PACS number(s): 05.10.Gg , 05.20.-y

\section{INTRODUCTION}

Recently, the Tsallis thermostatistics has received considerable attention in light of its growing application to a variety of physical systems [1]. The research has focused both on fundamental and phenomenological aspect of the issue [2].

Particular attention has been devoted to the issue of anomalous diffusion, where a significant experimental evidence has been gathered (see Ref. [3] for a detailed bibliography). The description of a diffusive process (either classic or anomalous) is performed generally by adopting a time dependent formalism. The Tsallis distribution namely

$$
p(v)=\frac{1}{Z_{q}}\left[1-(1-q) \beta v^{2}\right]^{1 /(1-q)},
$$

with $Z_{q}=\int_{\mathcal{R}} d v\left[1-(1-q) \beta v^{2}\right]^{1 /(1-q)}$, has been first derived starting from the generalized entropy

$$
S_{q}=\frac{1}{q-1}\left[1-\int_{\mathcal{R}} d v p^{q}\right]
$$

using the maximum entropy principle under the constraint of conservation of particle number and energy, by solving the variational problem: $\delta\left[S_{q}-\beta E-\alpha N\right]=0$.

Similarly to the classic Boltzmann distribution, the Tsallis distribution can be also obtained as the steadystate distribution of a time dependent Fokker-Planck
(FP) equation. Recently, the research on the derivation of the Tsallis distribution from FP equations has produced considerable results [3-13]. The research in this area can be classified in one of two classes.

First, linear FP equations are considered with diffusion and drift coefficients depending on the velocity. The dependence is chosen to lead to the Tsallis distribution as the equilibrium solution of the FP equation. Within the linear approach, two different choices of the drift and diffusion coefficients have been proposed. Stariolo [4] chooses a constant diffusion coefficients and alters the drift coefficient to include a generalized potential depending on the Tsallis parameter $q$. This approach introduces a more relevant modification of the classic Browninan approach. In ref. [5], instead, the classic Brownian drift coefficient have been considered, but with a modified diffusion coefficient to include a quadratic velocity dependence. The two linear approaches described above are in reality just examples of an infinite class of linear FP models that give a Tsallis equilibrium distribution [6]. Clearly, the selection of a specific linear model among the class requires the introduction of other criteria beyond the simple requirement of leading to an equilibrium Tsallis distribution.

Second, non-linear FP have been shown to lead to equilibrium Tsallis distributions. This approach, introduced by Plastino and Plastino [7] and continued by various authors [3,8-13], introduces a diffusion coefficient depending on powers of the distribution function. The drift, instead, can be equal to zero or described as in the classic Brownian motion. This latter approach, besides its elegance and simplicity, admits time dependent solutions characterized by retaining at every time the form of a Tsallis distribution. This self-similarity of the evolution represent an important property of the non-linear approach.

The present paper deals with the question of whether the linear [5] and non-linear [7] FP approach to the derivation of the Tsallis distributions are equivalent. The answer proved here is that indeed the two approaches are equivalent, in the sense that they both allow the presence of self-similar transients where the system is characterized by the Tsallis distribution at every instant.

In order to explain the microscopic origin of the anomalous diffusion associated to the non-linear FP equation of ref. [7] Borland suggested a feedback from the macroscopic level to the microscopic one [13]. In the present work, we show that the non-linear FP equation 
of ref. [7] but also the well known in the literature nonlinear porous media equation, considered recently in the frame of Tsallis thermostatistics in ref. [8], can be recast in the equivalent linear FP equation of ref. [5]. This important result allows a deeper interpretation of the nonlinear FP equation describing the anomalous diffusion in terms of a linear Langevin microdynamics in presence of a multiplicative noise.

The present work is organized as follows. In Section II, a Generalized Brownian (GB) motion is derived from the Langevin equation in presence of multiplicative noise. In Section III, the GB motion is shown to lead to a macroscopic motion described by the linear FP equation of ref. [5] that admits as a solution a class of time dependent Tsallis statistical distributions. In Section IV and V, the same distribution are shown to represents states governed also by the non linear FP equation of ref. [7] and by the non-linear porous media equation respectively. Finally, in Section VI conclusions are drawn.

\section{GENERALIZED BROWNIAN MOTION}

We consider the microscopic process described by the following Langevin equation:

$$
\frac{d v(t)}{d t}+h(t, v)=g(t, v) \Gamma(t)
$$

with

$$
\begin{gathered}
<\Gamma(t)>=0 \\
<\Gamma(t) \Gamma\left(t^{\prime}\right)>=2 \delta\left(t-t^{\prime}\right) .
\end{gathered}
$$

The quantity $-m h(t, v)$ is the deterministic force acting on a particle of mass $m$ and velocity $v(t)$ while $m g(t, v) \Gamma(t)$ is a stochastic force acting on the particle, with $\Gamma(t)$ a Gaussian random variable with zero mean and delta correlation function. The presence of $g(t, v)$ in eq. (1) implies that the particle is subject to a multiplicative noise. The distinction between additive (when $g(t, v)=$ const.) and multiplicative noise (when $g(t, v) \neq$ const. $)$ is very significant when $g(t, v)$ is a time dependent function. In this case arise naturally the question related to the definition of the stochastic integral (Ito or Stratonovich definition). For a more detailed discussion on multiplicative noise, see ref. [14]. The microscopic process described by eq. (1) implies a macroscopic process described by the following linear FP equation:

$$
\begin{aligned}
\frac{\partial p(t, v)}{\partial t}=\frac{\partial}{\partial v}\{[J(t, v) & \left.+\frac{\partial D(t, v)}{\partial v}\right] p(t, v) \\
& \left.+D(t, v) \frac{\partial p(t, v)}{\partial v}\right\},
\end{aligned}
$$

where the drift coefficient $J(t, v)$ and the diffusion coefficient $D(t, v)$ have the following expression

$$
\begin{gathered}
J(t, v)=h(t, v), \\
D(t, v)=g(t, v)^{2},
\end{gathered}
$$

obtained using the Ito definition for the stochastic integral. Note that, for Brownian motion:

$$
\begin{aligned}
& J(t, v)=\gamma(t) v \\
& D(t, v)=c(t)
\end{aligned}
$$

the drift current in eq.(4)

$$
j_{d r i f t}=\left[J(t, v)+\frac{\partial D(t, v)}{\partial v}\right] p(t, v),
$$

is simplified as:

$$
j_{d r i f t}=\gamma(t) v p(t, v)
$$

and the current velocity $j_{d r i f t} / p$ becomes simply proportional to the viscous force $-m h(t, v)=-m \gamma(t) v$ of the microscopic process.

A problem arises in conjunction to the results just obtained. Whether other motions, besides the Brownian motion, are characterized by a current velocity proportional to the viscous force. This issue corresponds to the existence of other solutions of the following equation for the unknown functions $D(t, v)$ and $J(t, v)$

$$
J(t, v)+\frac{\partial D(t, v)}{\partial v}=\theta(t) J(t, v)
$$

in addition to the solution (7),(8), relative to the Brownian motion. The issue is easily resolved and other solutions can be founded. The more general solution is formed by copies of functions $J(t, v)$ and $D(t, v)$ where $D(t, v)$ is given by

$$
D(t, v)=c(t)+[\theta(t)-1] \int J(v) d v,
$$

while $J(t, v)$ remains arbitrary. The simplest solution, for which $J(t, v)$ is given by (7) provides the definition for a new generalized Brownian (GB) motion [5].

\section{LINEAR FOKKER-PLANCK EQUATION}

We consider the FP equation (4) for the GB processes. With the introduction of the dimensionless time $\tau$ :

$$
d \tau=\theta(t) \gamma(t) d t
$$

and the functions $D(\tau), \beta(\tau)$ and parameter $q$ : 


$$
\begin{gathered}
D(\tau)=\frac{c(t)}{\theta(t) \gamma(t)}, \\
(1-q) \beta(\tau)=\frac{1-\theta(t)}{2 c(t)},
\end{gathered}
$$

the diffusion coefficient (12) with drift coefficient given by (7) can be written in the following form

$$
D(\tau, v)=D(\tau)\left[1-(1-q) \beta(\tau) v^{2}\right],
$$

while after taking into account (7), the FP equation (4) becomes [5]:

$$
\begin{aligned}
& \frac{\partial p(\tau, v)}{\partial \tau}=\frac{\partial}{\partial v}\{v p(\tau, v)+D(\tau) \\
& \left.\times\left[1-(1-q) \beta(\tau) v^{2}\right] \frac{\partial p(\tau, v)}{\partial v}\right\} .
\end{aligned}
$$

The time-dependent solutions of eq.(17) are sought using the following ansatz:

$$
p(\tau, v)=\frac{1}{Z_{q}(\tau)}\left[1-(1-q) \beta(\tau) v^{2}\right]^{1 /(1-q)} .
$$

The above ansatz requires the solution to conserve at every time the form of a Tsallis distribution with time dependent parameters $Z_{q}$ and $\beta$. The time dependence of the two parameters determines the actual solution and is obtained easily substituting ansatz (18) in eq.(17). It follows that the equations determining the evolution of $Z_{q}(\tau)$ and $\beta(\tau)$ are identical to the equations for the Brownian motion:

$$
\begin{gathered}
\frac{Z_{q}(\tau)}{Z_{q}(0)}=\left[\frac{\beta(0)}{\beta(\tau)}\right]^{1 / 2}, \\
\frac{d \beta(\tau)}{d \tau}=2 \beta(\tau)-4 D(\tau) \beta(\tau)^{2} .
\end{gathered}
$$

The result above justifies the use of the term Generalized Brownian motion used to name the process defined by eqs. $(7,12)$. From eq. (20) the condition below follows

$$
2 \beta(\infty) D(\infty)=1
$$

again in complete similarity with Browinan motion. Eq. (20) is solved easily with the the substitution $y=\beta^{-1}$ that linearizes the equation:

$$
\beta(\tau)=\beta(\infty)\left\{1+\left[\frac{\beta(\infty)}{\beta(0)}-1+a(\tau)\right] \exp (-2 \tau)\right\}^{-1},
$$

with

$$
a(\tau)=2 \int_{0}^{\tau}\left[\frac{D(\tau)}{D(\infty)}-1\right] \exp (-2 \tau) d \tau
$$

From eq. (19) it follows

$$
Z_{q}(\tau) \beta(\tau)^{1 / 2}=Z_{q}(0) \beta(0)^{1 / 2}=N_{q} .
$$

The constant $N_{q}$ is determined starting from the expression of $Z_{q}(\tau)$ given by

$$
Z_{q}(\tau)=\int_{-\infty}^{+\infty} d v\left[1-(1-q) \beta(\tau) v^{2}\right]^{1 /(1-q)}
$$

For $q \geq 1[15]$, it results:

$$
N_{q}=\frac{q+1}{2} \sqrt{\frac{q-1}{\pi}} \frac{\Gamma[1 / 2+1 /(q-1)]}{\Gamma[1 /(q-1)]} .
$$

The final solution of eq. (17) has the form

$$
p(\tau, v)=N_{q} \beta(\tau)^{1 / 2}\left[1-(1-q) \beta(\tau) v^{2}\right]^{1 /(1-q)},
$$

where $\beta(\tau)$ is given by eqs. (22-23).

\section{NON LINEAR FOKKER-PLANCK EQUATION}

The scope of the present and the next section is to show that the time dependent solution (27) obtained here, of the linear FP equation (17) proposed in [5], is also solution of non linear FP equations which can be obtained from the linear FP (17). The goal of the present section is to investigate the relationship between the linear FP (17) and the non-linear FP equation proposed by Plastino and Plastino [7].

We start the proof by noting that eq. (27) allows us to write

$$
1-(1-q) \beta(\tau) v^{2}=N_{q}^{q-1} \beta(\tau)^{(q-1) / 2} p(\tau, v)^{1-q} .
$$

Besides the following time dependent function is defined:

$$
D_{1}(\tau)=\frac{N_{q}^{q-1}}{2-q} D(\tau) \beta(\tau)^{(q-1) / 2}
$$

Then, it follows that eq.(17) can be rewritten as:

$$
\frac{\partial p(\tau, v)}{\partial \tau}=\frac{\partial}{\partial v}\left\{v p(\tau, v)+D_{1}(\tau) \frac{\partial}{\partial v}[p(\tau, v)]^{2-q}\right\}
$$

that is identical to the equation proposed by Plastino and Plastino that was solved using the same ansatz (18) used above but assuming that $D_{1}(\tau)$ is constant.

The procedure outlined in the previous section leads to the same relationship (19) between $Z_{q}(\tau)$ and $\beta(\tau)$ and $\beta(\tau)$ is governed by the following evolution equation:

$$
\frac{d \beta(\tau)}{d \tau}=2 \beta(\tau)-2 \beta(\infty)^{(q-3) / 2} \frac{D_{1}(\tau)}{D_{1}(\infty)} \beta(\tau)^{(5-q) / 2} .
$$

The condition (21) transforms now as 


$$
2 \beta(\infty)^{(3-q) / 2} D_{1}(\infty)=\frac{N_{q}^{q-1}}{2-q}
$$

As above, a transformation $y=\beta^{(q-3) / 2}$ linearizes eq.(31) and the general solution follows easily:

$$
\begin{gathered}
\beta(\tau)=\beta(\infty)\left(1+\left\{\left[\frac{\beta(\infty)}{\beta(0)}\right]^{(3-q) / 2}-1\right.\right. \\
\left.\left.+b_{q}(\tau)\right\} \exp [(q-3) \tau]\right)^{2 /(q-3)}, \\
b_{q}(\tau)=(3-q) \int_{0}^{\frac{(3-q) \tau}{2}}\left[\frac{D_{1}(\tau)}{D_{1}(\infty)}-1\right] \exp [(q-3) \tau] d \tau .
\end{gathered}
$$

The complete solution of eq. (30) is given by eq. (27) where now $\beta(\tau)$ is expressed as a function of $D_{1}(\tau)$ by eqs. (33-34). In the special case of $D_{1}(\tau)$ constant, the results presented in the literature $[3,7]$ are recovered.

\section{NON LINEAR POROUS MEDIA EQUATION}

For the solutions (27) considered above, the following results follows readily:

$$
v p(\tau, v)=\frac{N_{q}^{q-1}}{2(q-2)} \beta(\tau)^{(q-3) / 2} \frac{\partial}{\partial v}[p(\tau, v)]^{2-q} .
$$

Substituting the relationship above in eq.(30) it follows:

$$
\frac{\partial p(\tau, v)}{\partial \tau}=D_{2}(\tau) \frac{\partial^{2}}{\partial v^{2}}[p(\tau, v)]^{2-q},
$$

where

$$
D_{2}(\tau)=\frac{N_{q}^{q-1}}{q-2} \beta(\tau)^{(q-3) / 2}\left[\frac{1}{2}-\beta(\tau) D(\tau)\right] .
$$

As a consequence, eq. (36) is the well known non-linear porous media equation, widely used in condensed matter physics and considered recently in ref. [8]. The current of particles is given by:

$$
j(\tau, v)=-D_{2}(\tau) \frac{\partial}{\partial v}[p(\tau, v)]^{2-q},
$$

which generalizes the Fick law (indeed, for $q=1$ the classic Fick law is recovered).

Clearly, the time dependent Tsallis distribution (18) is solution of eq. (36) as well. $Z_{q}(\tau)$ and $\beta(\tau)$ are connected by eq. (19) and the solution acquires the form (27) while the evolution law for $\beta(\tau)$ follows from $D_{2}(\tau)$. The final differential equation is

$$
\frac{d \beta(\tau)}{d \tau}=4(q-2) N_{q}^{1-q} D_{2}(\tau) \beta(\tau)^{(5-q) / 2}
$$

that is solved readily to obtain

$$
\begin{aligned}
\beta(\tau) & =\beta(0)\left[1+2(q-2)(q-3) N_{q}^{1-q}\right. \\
& \left.\times \beta(0)^{(3-q) / 2} \int_{0}^{\tau} D_{2}(\tau) d \tau\right]^{2 /(q-3)}
\end{aligned}
$$

From eq. (37) and (39) it follows that $D_{2}(\infty)=0$, a condition that guarantees that the current (38) vanishes for $\tau \rightarrow \infty$, as needed to obtain an equilibrium state.

\section{CONCLUSIONS}

In the present work, the same time dependent Tsallis statistical distribution given in eq. (27) is solution of all three equations considered, eq. (17), eq.(30) and eq. (36) and describes anomalous diffusion. The time evolution of $\beta(\tau)$ is the same in all the cases considered and can be expressed in terms of the three functions $D(\tau), D_{1}(\tau)$, $D_{2}(\tau)$ that are inter-related.

The relationships derived above allow us to interpret the states (27) at a microscopic level. Such states are solutions of non linear FP equations (eqs.(30) and (36)) and consequently describe anomalous diffusion (when $q \neq 1$ ). But the results obtained above, prove that anomalous diffusion can also be described with the linear FP equation (17), which has a variable diffusion coefficient given by eq. (16).

The primary results of this equivalence is that the linear FP equation (17) can be related directly to the microscopic dynamic model expressed by the Langevin equation (1) linking in this way macroscopic processes described by anomalous diffusion with microscopic processes characterized by multiplicative noise.

[1] C. Tsallis, J. Stat. Phys., 52, 479 (1988).

[2] An updated bibliography on the Tsallis thermostatistics can be found in http://tsallis.cat.cbpf.br/biblio.htm

[3] C. Tsallis, D.J. Buckman, Phys. Rev. E, 54, R2197 (1996).

[4] D.A. Stariolo, Phys. Lett. A, 185, 262 (1994).

[5] G. Kanidakis, P. Quarati, Physica A, 237, 229 (1997).

[6] L. Borland, Phys. Lett. A, 245, 67 (1998).

[7] A.R. Plastino, A. Plastino, Physica A, 222, 347 (1995).

[8] A. Compte, D. Jou, J. Phys. A, 29, 4321 (1996).

[9] D.A. Stariolo, Phys. Rev. E, 55, 4806 (1997).

[10] A. Compte, D. Jou, Y. Katayama, J. Phys. A, 30, 1023 (1997).

[11] S. Martinez, A.R. Plastino, A. Plastino, Physica A, 259, 183 (1998). 
[12] L. Borland, F. Pennini, A.R. Plastino, and A. Plastino, Eur. Phys. J. B 12, 285 (1999).

[13] L. Borland, Phys. Rev. E, 57, 6634 (1998).

[14] H. Risken, The Fokker-Planck equation, (SpringerVerlag, Berlin Heidelberg 1989).

[15] C. Tsallis, S.V.F. Levy, A.M.C. Sousa, and R. Maynard, Phys. Rev. Lett. 75, 3589 (1995). 